\title{
A Lower Bound on the Distortion of Embedding Planar Metrics into Euclidean Space*
}

Ilan Newman and Yuri Rabinovich

Computer Science Department, University of Haifa,

Haifa 31905, Israel

\{ilan,yuri\}@cs.haifa.ac.il

\begin{abstract}
We exhibit a simple infinite family of series-parallel graphs that cannot be metrically embedded into Euclidean space with distortion smaller than $\Omega(\sqrt{\log n})$. This matches Rao's [14] general upper bound for metric embedding of planar graphs into Euclidean space, thus resolving the question how well do planar metrics embed in Euclidean spaces?
\end{abstract}

\section{Introduction}

Some of the most interesting questions in the study of finite metric spaces are about the relations between the structural properties of the underlying graph and of its geodetic metric (i.e., its shortest-path distance). In this paper we address one such question, and show a tight lower bound on the distortion of embedding a metric coming from a planar graph into Euclidean space.

Here are some basic definitions. A finite (semi-) metric space $(S, \mu)$ is a finite set $S$ and a symmetric nonnegative distance function $\mu$ on $S \times S$ satisfying the triangle inequality and $\mu(x, x)=0$. In what follows, we sometimes refer to $\mu$ as metric, regardless of $S$. A metric is called planar if it can be obtained by restricting the geodetic (i.e., shortest-path) metric of some weighted planar graph to a subset of its vertices. The weights should, of course, be nonnegative. Series-parallel, tree, etc., metrics are defined similarly. For example, one can easily check that the geodetic metric of the unit-weighted $K_{5}$ is a tree metric, while that of unit-weighted $K_{3,3}$ is not a planar metric.

* This research was supported in part by BSF Grant 1999325. 
Given two metric spaces $(S, \mu),(R, \delta)$ and an embedding $f: S \longrightarrow R$, the distortion of $f$ is defined as

$$
\operatorname{distr}(f)=\max _{x, y \in S} \frac{\delta(f(x), f(y))}{\mu(x, y)} \cdot \max _{x, y \in S} \frac{\mu(x, y)}{\delta(f(x), f(y))},
$$

i.e., the product of the maximum expansion and the maximum contraction of $f$. Observe that the distortion is never less than 1 , and is equal to 1 exactly when $f$ preserves $\mu$ up to scaling.

Two important parameters of a finite metric $\mu$ are $c_{2}(\mu)$ and $c_{1}(\mu)$, the smallest possible distortion of embedding $\mu$ into real Euclidean and $\ell_{1}$ space, respectively. Although the fundamental structural properties of finite metrics have just begun to emerge, numerous new exciting conjectures, theorems and applications (see, e.g., [8], [4], [10], [2], [3], [12], [6], [14], and [7]) seem to indicate that $c_{1}(\mu)$ and $c_{2}(\mu)$ indeed do capture some nontrivial aspects of $\mu$.

We summarize the relevant facts about these two parameters. It always holds that $c_{2}(\mu) \geq c_{1}(\mu)$, since any Euclidean metric is $\ell_{1}$-embeddable [13]. Assuming $\mu$ is a metric on $n$ points, both parameters are at most $O(\log n)$ [4], which is tight. The bound it is attained at the geodetic metric of any unit-weighted constant degree expander graph on $n$ points [10], [1].

Tree metrics behave significantly better: $c_{1}(\mu)$ is 1 , while $c_{2}(\mu)=O(\sqrt{\log \log n})$ [5], [12], which is attained at the geodetic metric of the unit-weighted full binary tree of depth $\log n$ [4], [12]. A natural next question is what happens in the case when $\mu$ is a planar metric, or, more generally, a metric coming from a graph excluding some fixed minor? An elegant result of Rao [14] using [9], shows that for such metrics $c_{2}(\mu)$, and consequently $c_{1}(\mu)$, are at most $O(\sqrt{\log n})$.

What are the true values of $c_{1}(\mu)$ and $c_{2}(\mu)$ for planar metrics? There are reasons to believe that $c_{1}(\mu)$ is bounded by a constant; see [7] for a related discussion, and a proof that this is indeed the case for series-parallel and outerplanar metrics. For the last two years it was not clear whether $c_{2}(\mu)$ is closer to $O(\sqrt{\log n})$, as in the upper bound of [14], or to $O(\sqrt{\log \log n})$, as for trees [12].

In this paper we settle this question, and establish a lower bound of $\Omega(\sqrt{\log n})$ already for series-parallel metrics, which are a special case of planar metrics.

In addition to the main result, we obtain as a by-product of our construction an infinite family of weighted planar (in fact, tree-width 3) graphs $H_{n}$, so that not only the entire geodetic metric of $H_{n}$, but even the lengths of its edges must be distorted by a factor of $\Omega(\sqrt{\log n})$ by any embedding of $H_{n}$ into Euclidean space. This contrasts with a construction of [11] showing that any weighted graph of tree-width 2 can be embedded into a line (!) so that the edges neither expand nor contract by more than a factor of 3, and also with a theorem of Seymour [15] about the existence of edge-preserving metric embeddings of weighted planar graphs into $\ell_{1}$ space.

\section{The Lower Bound}

Define a family $\left\{G_{k}\right\}$ of graphs in the following inductive manner, similar to the one used in [7]: 


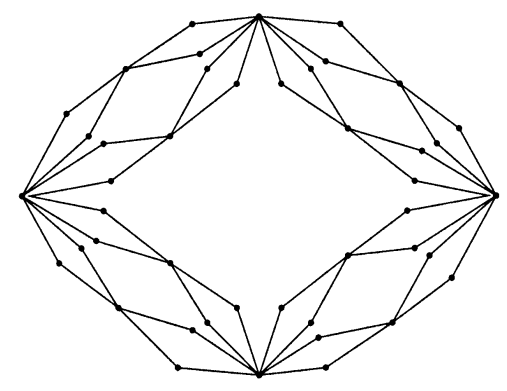

Fig. 2.1. The graph $G_{3}$.

$G_{0}$ consists of a single edge. $G_{i}$ is a "refinement" of $G_{i-1}$ obtained by replacing each edge of $G_{i-1}$ by two parallel paths, each containing two edges. See Fig. 2.1. The length of every edge in $G_{i}$ is defined as $2^{-i}$, half that of $G_{i-1}$. It is convenient to identify $V\left(G_{i-1}\right)$ with the "old" vertices of $G_{i}$. Observe that this natural identification is in fact an isometry, i.e., the restriction of the geodetic metric of $G_{i}$ to the "old" vertices is identical with the geodetic metric of $G_{i-1}$ on these vertices.

Thus, without risk of confusion, we can speak of edges of $G_{i}$, and of their length, while discussing any $G_{k}, k \geq i$. To simplify the presentation, we also introduce the notion of an anti-edge. Assume that the edge $(a, b)$ of $G_{i-1}$ was replaced in $G_{i}$ by edges $(a, x),(x, b)$ and $(a, y),(y, b)$, respectively. The pair of vertices $\{x, y\} \subset V\left(G_{i}\right)$ will be called the anti-edge of $(a, b)$. Observe that the distance between $x$ and $y$ is $2^{-i}$, exactly as the length of the edge $(a, b)$ of $G_{i-1}$.

It is easily checked that $G_{k}$ is a series-parallel graph containing $4^{k}$ edges and $\left(2 \cdot 4^{k}\right.$ $+4) / 3$ vertices.

Theorem 2.1. Let $\mu$ denote the geodetic metric of $G_{k}$. Then

$$
c_{2}(\mu) \geq \sqrt{k+1} \text {. }
$$

Proof. Let $f: V(G) \longrightarrow \mathbb{R}^{d}$ be an embedding of $\mu$ into Euclidean space. Due to the scalability of Euclidean space, we may without loss of generality assume that $f$ is nonexpanding, i.e., $\|f(v)-f(u)\|_{2} \leq \mu(u, v)$ for any $u, v \in V\left(G_{k}\right)$. Let $\alpha=$ $\min _{v, u \in V\left(G_{k}\right)}\left(\|f(v)-f(u)\|_{2} / \mu(v, u)\right)$. Our goal will be to show that $\alpha \leq 1 / \sqrt{k+1}$, i.e., some distance must contract under such $f$ by at least $\sqrt{k+1}$.

First, we prove by downwards induction on $i$ that the length of any edge of $G_{i}$, $i=k, k-1, \ldots, 0$, must contract under $f$ by at least a factor of $\left[1-(k-i) \alpha^{2}\right]^{-1 / 2}$. Formally, we claim that for any $(a, c) \in E\left(G_{i}\right)$,

$$
\|f(a)-f(c)\|_{2} \leq \sqrt{1-(k-i) \alpha^{2}} \cdot \mu(a, c) .
$$

The claim is trivially true for $i=k$, since $f$ is nonexpanding. Assume we have already demonstrated the claim for $i+1$; we demonstrate it for $i$. Recall the well known inequality that for any four points $a, b, c, d$ in Euclidean space the sum of the squares of the diagonals never exceeds the sum of the squares of the sides:

$$
\|a-b\|_{2}^{2}+\|b-c\|_{2}^{2}+\|c-d\|_{2}^{2}+\|d-a\|_{2}^{2} \geq\|a-c\|_{2}^{2}+\|b-d\|_{2}^{2} .
$$


The inequality holds since after subtracting the right-hand side from the left-hand side, one arrives at $\|a-b+c-d\|_{2}^{2}$.

Now, consider an edge $(a, c)$ of $G_{i}$, its anti-edge $(b, d)$ and the four surrounding edges of $G_{i+1}:(a, b),(b, c),(c, d)$ and $(d, a)$. By our inductive assumption, the images of each of the latter pairs are at most $\sqrt{1-(k-i-1) \alpha^{2}} \cdot 2^{-(i+1)}$ apart. By the definition of $\alpha$, the images of $b$ and $d$ are at least $\alpha \cdot 2^{-i}$ apart. Combining this with (2.1), we get

$$
4 \cdot 2^{-2(i+1)} \cdot\left[1-(k-i-1) \alpha^{2}\right] \geq \alpha^{2} \cdot 2^{-2 i}+\|f(a)-f(c)\|_{2}^{2},
$$

from which we conclude that

$$
\|f(a)-f(c)\|_{2}^{2} \leq\left[1-(k-i) \alpha^{2}\right] \cdot 2^{-2 i}=\left[1-(k-i) \alpha^{2}\right] \cdot \mu^{2}(a, c),
$$

as claimed.

Consider the edge $(s, t)$ of $G_{0}$. The images of $s$ and $t$ are at least $\alpha$ apart by definition of $\alpha$, and at most $\sqrt{1-k \cdot \alpha^{2}}$ apart by the claim. Comparing the two terms we conclude that

$$
\alpha \leq 1 / \sqrt{k+1}
$$

Theorem 2.1 can be slightly strengthened. Observe that in our proof we have used only the edges and the anti-edges of $G_{i}$ 's. Therefore, restoring all these pairs as edges, and assigning them weight equal to their distance in $G_{k}$, we arrive at the graph $H_{k}$, whose edges must suffer distortion $\sqrt{k+1}$ in any embedding of $H_{k}$ into Euclidean space. Formally:

Let $H_{0}$ consist of single edge of length 1 , and let $H_{i}$ be obtained by taking $H_{i-1}$, and in addition to existing vertices and edges, introducing for each edge $e=(a, c)$ of $H_{i-1}$ of length $2^{-(i-1)}$ two new vertices $b_{e}, d_{e}$, a new edge $\left(b_{e}, d_{e}\right)$ of length $2^{-(i-1)}$ and four new edges $\left(a, b_{e}\right),\left(b_{e}, c\right),\left(c, d_{e}\right)$ and $\left(d_{e}, a\right)$, each of length $2^{-i}$. As before, $H_{i-1}$ isometrically embeds into $H_{i}$ under the natural identification of the vertices.

It turns out that $H_{k}$ is still planar, and, moreover, has tree-width 3 . It has $\left(5 \cdot 4^{k}-2\right) / 3$ edges and the same number of vertices as in $G_{k}$. By the preceding discussion we get

Theorem 2.2. In any embedding $f$ of $H_{k}$ into Euclidean space which does not expand the edges, there exists an edge in $E\left(H_{k}\right)$ whose length is contracted by $f$ by at least a factor of $\sqrt{k+1}$.

\section{Acknowledgments}

We are grateful to Anupam Gupta for mentioning to us to the construction of Rao, and clarifying it. Thanks also to Nati Linial and Jirka Matoušek for insightful discussions.

\section{References}

1. Y. Aumann and Y. Rabani. An $O(\log k)$ approximate min-cut max-flow theorem and approximation algorithm. SIAM Journal on Computing, 27(1):291-301, February 1998.

2. Y. Bartal. Probabilistic approximations of metric spaces and its algorithmic applications. In Proceedings of the 37th Annual IEEE Symposium on Foundations of Computer Science, pages 184-193, 1996. 
3. Y. Bartal. On approximating arbitrary metrics by tree metrics. In Proceedings of the 30th Annual ACM Symposium on Theory of Computing, pages 161-168, 1998.

4. J. Bourgain. On Lipschitz embeddings of finite metric spaces in Hilbert space. Israel Journal of Mathematics, 52(1-2):46-52, 1985.

5. J. Bourgain. The metrical interpretation of superreflexivity in Banach spaces. Israel Journal of Mathematics, 56(2):222-230, 1986.

6. U. Feige. Approximating the bandwidth via volume respecting embeddings. In Proceedings of the 30th Annual ACM Symposium on Theory of Computing, pages 90-99, 1998.

7. A. Gupta, I. Newman, Y. Rabinovich and A. Sinclair. Cuts, trees and $\ell_{1}$-embeddings of graphs. In Proceedings of the 40th Annual Symposium on Foundations of Computer Science, pages 399-408, 1999.

8. W. B. Johnson and J. Lindenstrauss. Extensions of Lipschitz mappings into a Hilbert space. Contemporary Mathematics 26:189-206, 1984.

9. P. Klein, S. A. Plotkin and S. B. Rao. Excluded minors, network decomposition, and multicommodity flow. In Proceedings of the 25th Annual ACM Symposium on Theory of Computing, pages 682-690, 1993.

10. N. Linial, E. London and Y. Rabinovich. The geometry of graphs and some of its algorithmic applications. Combinatorica, 15(2):215-245, 1995.

11. N. Linial, J. Matoušek and Y. Rabinovich. Embedding the edges of series-parallel graphs into line. Manuscript, 2000.

12. J. Matoušek. On embedding trees into uniformly convex Banach spaces. Israel Journal of Mathematics, 114:221-237, 1999. (Czech version in: Lipschitz distance of metric spaces, C.Sc. degree thesis, Charles University, 1990.)

13. V. Milman and G. Schechtman. Asymptomic Theory of Finite Dimensional Spaces, LNM 1200, SpringerVerlag, Berlin, 1986.

14. S. B. Rao. Small distortion and volume preserving embeddings for planar and Euclidean metrics. In Proceedings of the 15th Annual ACM Symposium on Computational Geometry, pages 300-306, 1999.

15. P. D. Seymour. Sums of circuits. In J. A. Bondy and U. S. Murty, editors, Graph Theory with Applications, pages 341-355. American Elsevier, New York, 1979.

Received April 4, 2001, and in revised form April 28, 2002. Online publication November 21, 2002. 